\title{
Comparación entre dos modelos estructurados por edades, aplicados a la pesquería de langosta, Panulirus argus (Latreille, 1804), en la región suroriental de Cuba
}

\section{Comparison between two age-structured models, applied to the spiny lobster Panulirus argus (Latreille, 1804) fishery in Cuba's southeastern region}

\author{
Romina Alzugaray Martínez $z^{*}$ y Rafael Puga Millán ${ }^{1}$
}

\begin{abstract}
RESUMEN
La langosta común, Panulirus argus, es una de las especies con mayor valor comercial en el Atlántico Centro Occidental. En Cuba se han realizado numerosos estudios para conocer y actualizar su estado de explotación y recomendar medidas de manejo. A pesar de estas medidas, las capturas continúan disminuyendo, por lo que el objetivo del presente estudio consistió en evaluar la dinámica de la población de langosta en la región suroriental de Cuba, a través de dos estrategias analíticas diferentes. A partir de datos de captura y esfuerzo pesquero de 1979-2010, se aplicaron un análisis de población virtual (VPA) y un análisis estadístico de captura por edades (SCA). Se examinó la relación lineal entre los datos primarios y las variables estimadas por los modelos. El ajuste de los modelos lineales de los datos se evaluó mediante el Criterio de Información de Akaike corregido $\left(\mathrm{AIC}_{\mathrm{c}}\right.$ ). Según ambos métodos de captura por edades, el tamaño poblacional y el reclutamiento de langostas con un año de edad han disminuido en la región en el período estudiado, aunque el SCA muestra estabilización en la última década. Mientras, la biomasa poblacional disminuyó hasta estabilizarse en la última década, lo cual puede relacionarse con el comportamiento histórico de la captura por unidad de esfuerzo. Existen asociaciones lineales significativas entre los datos primarios y las variables estimadas. Según los valores de $\Delta_{i}$, el modelo VPA garantiza el mejor ajuste de las variables a las relaciones lineales estimadas.
\end{abstract}

Palabras claves: Criterios de información, Cuba, modelos estructurados por edades, langosta, pesquería.

\begin{abstract}
Spiny lobster, Panulirus argus, is one of the most commercially valuable species in the Western Central Atlantic. Although numerous studies have been conducted in Cuba to learn and update its exploitation status and to recommend management measures, catches continue to decline. Consequently, the objective of this study was to evaluate the dynamics of the lobster population in Cuba's southeastern region, through two different analytical strategies. Using catch and fishing effort data from 1979-2010, a Virtual Population Analysis (VPA) and a Statistical Catch-at-age Analysis (SCA) were applied. We examined the linear relationship between raw data and the variables estimated by the models. The fit of the linear models to data was assessed using the corrected Akaike Information Criterion ( $\mathrm{AIC}_{\mathrm{c}}$ ). According to both age-structured methods, population size and recruitment of one year old lobster have declined in the region during the study period, although the SCA shows stabilization in the last decade. Population biomass decreased to stabilize in the last decade, this may relate to the historical behavior of the catch per unit effort. There are significant linear associations between raw data and estimated variables. According to $\Delta_{i}$ values, the VPA model ensures the best fit for the variables of estimated linear relationships.
\end{abstract}

Keywords: Age-structured models, Cuba, fishery, information criterion, spiny lobster.

1 Centro de Investigaciones Pesqueras, Calle 246 y 5ta Avenida, Barlovento, Santa Fe, Playa, Ciudad de La Habana, Cuba. CP.19100.romina@cip.telemar.cu*,rpuga@cip.telmar.cu

Recibido 13-XII-2011

Aceptado 13-VIII-2012

DOI: http://dx.doi.org/10.15359/revmar.4.9

Rev. Mar. Cost. ISSN 1659-455X. Vol. 4: 131-143, Diciembre 2012. 


\section{INTRODUCCIÓN}

La langosta común, Panulirus argus (Latreille, 1804), es una de las especies de mayor valor comercial en el Atlántico Centro Occidental (Ehrhardt et al. 2011). Alrededor de 26 países están involucrados en su pesquería y comercialización. Cuba, Bahamas y Brasil son los principales productores con más del $60 \%$ del total capturado en la región, seguidos por Estados Unidos, Honduras y Nicaragua (Ehrhardt, 2001).

En Cuba, la langosta es el recurso marino más importante. Los ingresos de esta pesquería se estiman en aproximadamente 80 millones de dólares estadounidenses por año. Además, miles de personas son empleadas en actividades extractivas e industriales relacionadas con esta pesquería (Baisre, 2000).

Debido a la importancia de esta pesquería en Cuba, se han realizado numerosos estudios para conocer y actualizar el estado de explotación de la langosta y recomendar medidas de manejo adecuadas con su situación (de León et al. 1991; Puga et al. 2005; 2010).

En las evaluaciones de los stocks de especies comerciales con abundantes datos, algunos de los métodos más usados son los modelos de captura por edades de una sola especie. Estos modelos permiten la reconstrucción histórica del número de individuos por edades de los stocks explotados y proporcionan estimaciones de las principales tasas de mortalidad a la que la especie extraída se encuentra sujeta (Jurado-Molina et al. 2005). Estas estimaciones sirven como base principal para proveer medidas de manejo en muchas pesquerías alrededor del mundo (Megrey, 1989). Se reconocen dos tipos principales de métodos de captura por edades: el análisis de población virtual (VPA) y el análisis estadístico de captura por edades (SCA) (Hilborn \& Walters, 1992; Haddon, 2001). Las variables poblacionales estimadas (tamaño de la población, biomasa, tasa de mortalidad, entre otras) dependen de la calidad del sistema de control y los métodos utilizados para analizar los datos, por lo tanto, antes de la adopción de un conjunto de indicadores y de otros resultados de la evaluación de stock, se recomienda evaluar si los datos y métodos analíticos utilizados pueden proporcionar estimaciones razonables de estas cantidades (Punt et al. 2002).

Para el manejo de esta pesquería se han tomado numerosas medidas reguladoras. Entre las principales se encuentran las siguientes: régimen de propiedad estatal, acceso controlado y limitado a la pesquería, división territorial por empresas y por microzonas, veda de cuatro y medio meses para proteger los principales picos de desove y de reclutamiento de preadultos al área de pesca, talla mínima legal de $76 \mathrm{~mm}$ de largo de cefalotórax (LC), prohibición de pescar en áreas de cría, prohibición de desembarcar hembras con cualquier señal externa de actividad reproductiva, entre otras (Puga et al. 2010).

A pesar de estas medidas reguladoras, las capturas continúan disminuyendo, y al ser la región suroriental la segunda en importancia (Alzugaray \& Puga, 2010), el objetivo del presente estudio consistió en evaluar la dinámica de la población de langosta espinosa en la región, a través de dos estrategias analíticas diferentes.

\section{MATERIALES Y MÉTODOS}

\section{Zona de estudio}

La plataforma suroriental de Cuba tiene una extensión total de $18800 \mathrm{~km}^{2}$, y el área de pesca de langosta constituye el 30\% (5 $702 \mathrm{~km}^{2}$ ) de esta (Cruz et al. 1990) (Fig. 1).

\section{Fuente de datos}

Los datos primarios son: captura de langosta (C) en toneladas, esfuerzo pesquero (f), como el total de días de pesca de todas las embarcaciones en la zona durante el año, y la clasificación industrial por grupos 


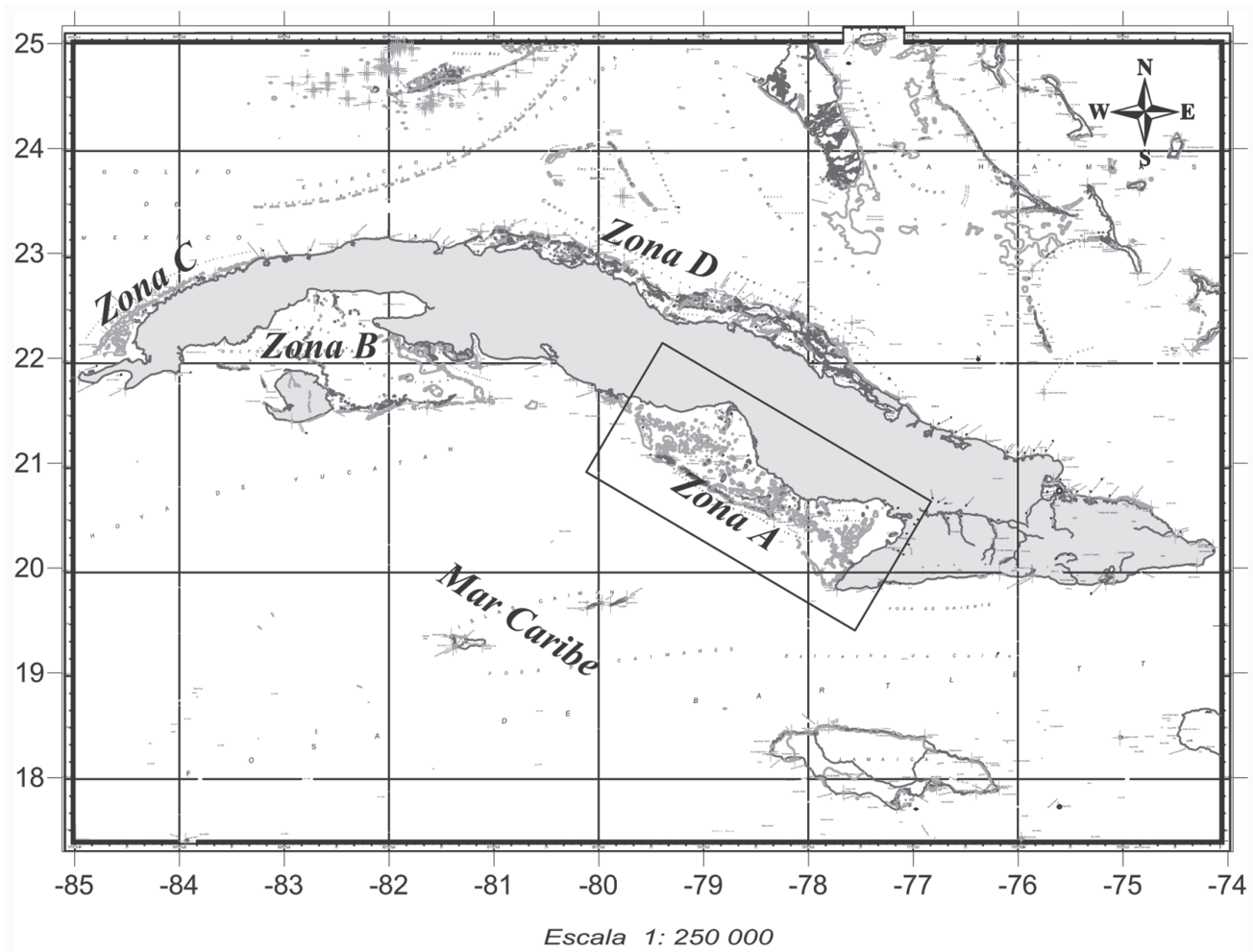

Fig. 1. Localización de las zonas de pesca en la plataforma cubana. El recuadro indica la región suroriental de Cuba, zona de estudio (Zona A)

Fig. 1. Location of fishing zones in the Cuban plataform. The box indicates Cuba's southeastern region, study area (Zone $\mathrm{A})$

de peso, se adquirieron a partir de las estadísticas de las empresas pesqueras que operan en la plataforma suroriental. Se procesaron los valores obtenidos para el período comprendido entre los años 1979-2010.

\section{Parámetros}

La tasa de mortalidad natural (M) se estimó como $\mathrm{M}=0.34$ año $^{-1}$ a partir de la ecuación empírica propuesta por Cruz et al. (1981), que relaciona la mortalidad natural de varias especies de langosta con la temperatura media del agua $\left(\mathrm{T}=27^{\circ} \mathrm{C}\right)$ y los parámetros de crecimiento de estas. Para el cálculo de M se utilizaron los parámetros de crecimiento calculados por de León et al. (1995), considerados los más consistentes para la especie en el área (Arce \& de León, 2001).
Las conversiones entre la longitud o edad y el peso se realizaron empleando las constantes estimadas por Cruz et al. (1981) para la relación longitud-peso:

$$
\mathrm{W}=0.00243 \mathrm{LC}^{2.764}
$$

Donde W es el peso total (g) y LC es la longitud del cefalotórax ( $\mathrm{mm})$.

La producción de huevos se calculó a partir de las relaciones de la longitud del cefalotórax con la maduración y la fecundidad (Cruz \& de León, 1991), teniendo en cuenta que las hembras mayores de $80 \mathrm{~mm}$ de LC producen dos desoves anuales (Lipcius, 1985). Las frecuencias de tallas de las capturas se agruparon en intervalos de $5 \mathrm{~mm}$ de LC y, posteriormente, se convirtieron a 
edades mediante el método de rebanado propuesto por Sparre \& Venema (1995).

\section{Análisis de población virtual (VPA)}

A partir de la matriz de captura a las edades entre 1 y 9+ años, se aplicó un VPA mediante el método ADAPT (Gavaris, 1988) con la modificación propuesta por MacCall (1986), que permite mejores ajustes para valores de $\mathrm{M}>0.3$.

Para todas las edades del último año (2010) y la última edad de todos los años:

$$
\mathrm{N}_{\mathrm{a}, \mathrm{t}}=\frac{\mathrm{C}_{\mathrm{a}, \mathrm{t}}}{\frac{\mathrm{F}_{\mathrm{a}, \mathrm{t}}}{\mathrm{F}_{\mathrm{a}, \mathrm{t}}+\mathrm{M}}}\left(1-\mathrm{e}^{-\left(\mathrm{F}_{\mathrm{a}, \mathrm{t}}+\mathrm{M}\right)}\right)
$$

Para el resto de las edades y años:

$$
\begin{gathered}
\mathrm{N}_{\mathrm{a}, \mathrm{t}}=\left(\mathrm{N}_{\mathrm{a}+1, \mathrm{t}+1} \mathrm{e}^{\mathrm{M}}\right)+\mathrm{C}_{\mathrm{a}, \mathrm{t}}\left(\frac{\mathrm{M}}{1-\mathrm{e}^{-\mathrm{M}}}\right) \\
\mathrm{F}_{\mathrm{a}, \mathrm{t}}=\ln \left(\frac{\mathrm{N}_{\mathrm{a}, \mathrm{t}}}{\mathrm{N}_{\mathrm{a}+1, \mathrm{t}+1}}\right)-\mathrm{M}
\end{gathered}
$$

Donde $\mathrm{N}_{\mathrm{a}, \mathrm{t}}$ es el número de individuos con edad a al inicio del año $t, \mathrm{C}_{\mathrm{a}, \mathrm{t}} \mathrm{y} \mathrm{F}_{\mathrm{a}, \mathrm{t}}$ son la captura y la mortalidad por pesca a la edad a durante el año t, y M es la mortalidad natural.

Los cálculos para obtener matrices por año y edad de $\mathrm{F}, \mathrm{N}$ y el número de huevos se realizaron según la metodología propuesta por Lassen \& Medley (2000).

La captura por unidad de esfuerzo (CPUE) por edades y años, expresada en número de langostas por días de pesca, se utilizó como índice de abundancia para ajustar el VPA. El ajuste se realizó minimizando una función objetivo, compuesta por la suma de cuadrados de los errores de los logaritmos de la matriz del índice de proporcionalidad entre el tamaño de la población (N) y el índice de abundancia por edades, en relación con el valor promedio a cada edad. Este índice se llama capturabilidad (q) y se expresa:

$$
\mathrm{q}_{\mathrm{a}, \mathrm{t}}=\frac{\mathrm{CPUE}_{\mathrm{a}, \mathrm{t}}}{\mathrm{N}_{\mathrm{a}, \mathrm{t}}}
$$

Donde CPUE es $_{\mathrm{t}}$ es la captura por unidad de esfuerzo, $\mathrm{q}_{\mathrm{a}, \mathrm{t}}$ es el coeficiente de capturabilidad y $\mathrm{N}_{\mathrm{a}, \mathrm{t}}$ es el número de langostas en la población, de edad a en el año t.

Entonces:

$$
\ln \left(\mathrm{q}_{\mathrm{a}, \mathrm{t}}\right)=\ln \left(\mathrm{CPUE}_{\mathrm{a}, \mathrm{t}}\right)-\ln \left(\mathrm{N}_{\mathrm{a}, \mathrm{t}}\right)
$$

Y la función objetivo queda como:

$$
\sum\left[\ln \left(q_{a, t}\right)-\ln \left(q_{a}\right)\right]^{2}
$$

Donde $\ln \left(\mathrm{q}_{\mathrm{a}}\right)$ es el valor promedio para cada edad a.

La optimización de la función objetivo se realizó variando la $\mathrm{F}$ de la edad 8 en el último año. Las F del grupo 9+ en cada año se asumen iguales a la edad 8, mientras que las $\mathrm{F}$ de las edades 1-7 para el último año fueron estimadas mediante la relación con la edad 8 durante el período 2007-2009.

\section{Análisis estadístico de captura por eda- des (SCA)}

A partir de la misma matriz de captura a las edades entre 1 y 9+ años, se aplicó un análisis estadístico de captura por edades (SCA), descrito por Haddon (2001).

Con el reclutamiento inicial, y la tasa máxima de mortalidad por pesca a la edad para cada año combinada con la mortalidad natural (M), se completó la matriz de número de individuos por edades:

$$
\begin{gathered}
\mathrm{N}_{\mathrm{a}+1, \mathrm{t}+1}=\mathrm{N}_{\mathrm{a}, \mathrm{t}} \mathrm{e}^{-\left(\mathrm{M}+\mathrm{F}_{\mathrm{a}, \mathrm{t}}\right)} \\
\mathrm{F}_{\mathrm{a}, \mathrm{t}}=\mathrm{F}_{\mathrm{t}} \mathrm{S}_{\mathrm{a}}
\end{gathered}
$$


Donde $\mathrm{N}_{\mathrm{a}, \mathrm{t}}$ es el número de langostas, $\mathrm{F}_{\mathrm{a}, \mathrm{t}}$ es la mortalidad por pesca, a la edad a durante el año t, $M$ es la mortalidad natural y $\mathrm{S}_{\mathrm{a}}$ es la selectividad a la edad a.

Para ajustar el modelo se calcularon diferentes funciones de verosimilitud:

$$
\mathrm{L}_{\mathrm{C}}=\frac{\mathrm{S}^{2}}{2} \sum\left(\ln \mathrm{C}_{\mathrm{a}, \mathrm{t}}-\ln \hat{\mathrm{C}}_{\mathrm{a}, \mathrm{t}}\right)^{2}
$$

para las capturas en número observadas y pronosticadas para cada edad a en cada año t.

$$
\mathrm{L}_{\mathrm{F}}=\frac{\mathrm{S}^{2}}{2} \sum\left(\ln \mathrm{F}_{\mathrm{t}}-\ln \hat{\mathrm{F}}_{\mathrm{t}}\right)^{2}
$$

para las mortalidades por pesca observadas y pronosticadas en cada año $t$.

$$
\mathrm{L}_{\mathrm{Y}}=\frac{\mathrm{S}^{2}}{2} \sum\left(\ln \mathrm{Y}_{\mathrm{t}}-\ln \hat{\mathrm{Y}}_{\mathrm{t}}\right)^{2}
$$

para las capturas observadas (ton) y pronosticadas en cada año t.

$\mathrm{L}_{\mathrm{CPUE}}=\frac{\mathrm{S}^{2}}{2} \sum\left(\operatorname{lnCPUE}_{\mathrm{t}}-\mathrm{CPUE}_{\mathrm{t}}\right)^{2}$

para las capturas por unidad de esfuerzo observadas y pronosticadas en cada año t.

$$
\mathrm{L}_{\mathrm{R}}=\frac{\mathrm{S}^{2}}{2} \sum\left(\ln \mathrm{R}_{\mathrm{t}}-\ln \hat{\mathrm{R}}_{\mathrm{t}}\right)^{2}
$$

para el reclutamiento "observado" y pronosticado en cada año t.

El ajuste se realizó minimizando una función objetivo compuesta por la suma total de las funciones de verosimilitud multiplicadas por el peso de cada función en el total.

La optimización de la función objetivo se realizó variando los estimados iniciales de reclutamiento y las tasas máximas de mortalidad por pesca.

\section{Análisis de Regresión Lineal}

Se examinó la relación lineal entre los datos primarios (captura, esfuerzo pesquero y captura por unidad de esfuerzo) y las variables estimadas $(\mathrm{N}, \mathrm{R}, \mathrm{B}$ y $\mathrm{F})$ por los modelos pesqueros (C vs. N, C vs. B, $\mathrm{C}$ vs. F, C vs. $\mathrm{R}_{(\mathrm{t}-2)}$, CPUE vs. N, F vs. f) mediante el análisis de regresión lineal, con un nivel de significación $P \leq 0.05$. Previamente, se comprobó que todas las variables tuvieran normalidad y homogeneidad de varianzas. Los análisis se ejecutaron con el programa STATISTICA 6.0 (StatSoft, 2001).

\section{Comparación entre modelos}

El ajuste de los modelos lineales a los datos para cada método de captura por edades se evaluó mediante el Criterio de Información de Akaike corregido ( $\mathrm{AIC}_{\mathrm{c}}$ ). Según Quinn \& Keough (2002), los criterios se calculan como:

$$
\mathrm{AIC}_{\mathrm{C}}=\mathrm{n} \ln \mathrm{SS}_{\text {residual }}+2(\mathrm{p}+1)+\mathrm{n} \ln \mathrm{n}
$$

Donde $\mathrm{SS}_{\text {residual }}$ es la suma de cuadrados de los residuales, $n$ es el tamaño de muestra y $\mathrm{p}$ se refiere al número de parámetros del modelo.

Para cada modelo se calculó la diferencia entre el valor correspondiente de $\mathrm{AIC}_{\mathrm{c}} \mathrm{y}$ el valor mínimo de $\mathrm{AIC}_{\mathrm{c}}$ obtenido en alguno de los modelos, i.e., $\mathrm{D}_{i}=\mathrm{AICc}_{\mathrm{i}}$ - $\mathrm{AICc}_{\min }$. Valores de $\mathrm{D}_{i}>2$ indican poco apoyo empírico para el modelo $i$. Este procedimiento es válido para modelos no jerárquicos (Burnham \& Anderson, 2002).

\section{RESULTADOS}

En la actualidad, la pesquería de langosta espinosa ( $P$. argus) en la región suroriental de Cuba se encuentra muy deprimida: las capturas han disminuido un $80 \%$ 


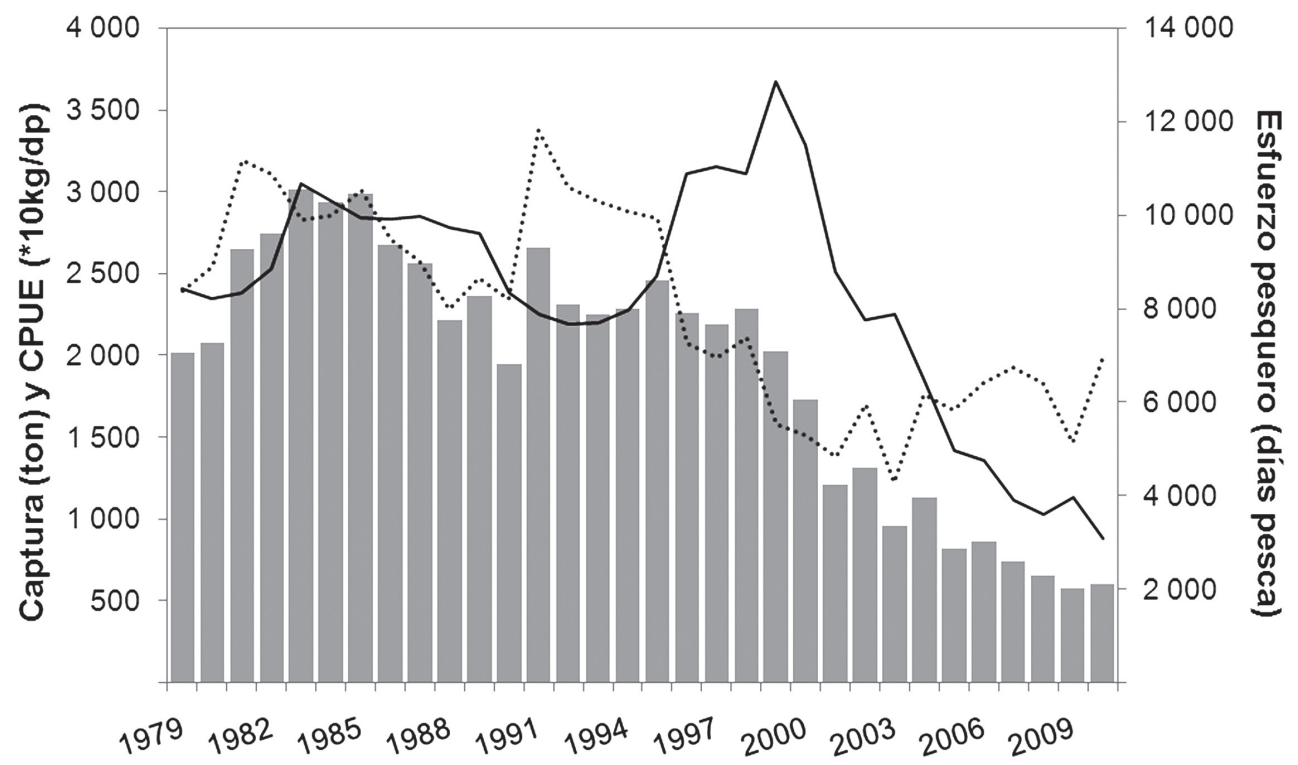

Fig. 2. Series anuales de captura (columnas), esfuerzo pesquero (línea sólida) y captura por unidad de esfuerzo (CPUE) (línea punteada) de la pesquería de langosta espinosa en la región suroriental de Cuba, entre 1979-2010

Fig. 2. Annual catch series (columns), fishing effort (solid line) and catch per unit effort (CPUE) (dotted line) of the spiny lobster fishery in Cuba's southeastern region, 1979-2010

(608 ton en el año 2010) desde mediados de los años 80, cuando se obtuvieron los máximos niveles (2 980 ton, promedio 1983-1985). Esto ha conllevado a la reducción del 72\% (3 071 días pesca en el año 2010) del nivel de esfuerzo pesquero aplicado, lográndose CPUE $(180 \mathrm{~kg} / \mathrm{días}$ pesca, promedio 2008-2010) que son solo la mitad de las alcanzadas en la época de máximo esplendor $(311 \mathrm{~kg} /$ días pesca, promedio 1983-1985) (Fig. 2).

Las tendencias del tamaño total de la población $(\mathrm{N})$, su biomasa (B), el reclutamiento (R) y la tasa de mortalidad por pesca (F) son consistentes entre los dos modelos matemáticos (VPA y SCA) en el período que se analiza (Fig. 3).

El tamaño total de la población de langosta para ambos modelos presenta una tendencia a la disminución, desde los máximos valores a principios de los años 80 (alrededor de 50 millones de langostas) hasta
16 millones de langostas en el año 2010, según el VPA, mientras el SCA muestra en la última década una tendencia a la estabilización de la población, alrededor de los 23 millones de individuos (Fig. 3A).

El reclutamiento de langostas con un año de edad a la población (R) muestra una tendencia muy similar al tamaño de la población $(\mathrm{N})$, con los máximos valores a principios de los 80 (alrededor de 17 millones de reclutas), disminuyendo hasta llegar a solo 4 millones de reclutas en el año 2010, según el modelo VPA. Por su parte, el modelo SCA muestra una tendencia similar, si bien en la última década, se aprecia una estabilización alrededor de los 8 millones de reclutas (Fig. 3B).

La biomasa total (B) de la población de langostas tiene una tendencia similar a la disminución, más marcada en los resultados del SCA, mientras que, según el VPA, se mantuvo más o menos estable en 
más de 14000 ton entre principios de los 80 y los 90 . En la última década, ambos modelos muestran una estabilización alrededor de las 8000 ton (Fig. 3C).

El estimado de la tasa de mortalidad por pesca $(F)$ a la que está sometida esta población tiene un comportamiento histórico muy similar, según ambos modelos, si bien la estimación del SCA es un 5\% mayor que la del VPA. Los mayores valores se obtuvieron a finales de los años 90 (alrededor de 0.25 (VPA) y 0.30 (SCA)) (Fig. 3D).

El valor medio de la captura (ton) es $1923.8 \pm 777.0$, del esfuerzo pesquero (días pesca) es $8250.6 \pm 2479.1$ y de la

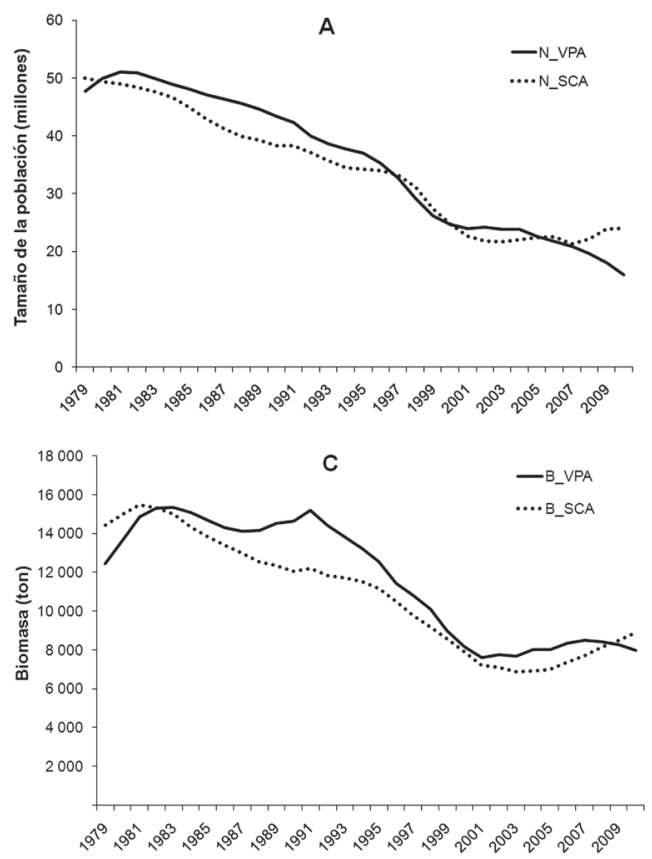

captura por unidad de esfuerzo $(\mathrm{kg} / \mathrm{días}$ pesca) es $229.2 \pm 60.5$.

Los valores promedios de las variables estimadas no presentan diferencias significativas entre ambos modelos $(P \geq$ 0.05) (Cuadro 1).

Las relaciones propuestas $(\mathrm{C} v s . \mathrm{N}, \mathrm{C}$ vs. B, C vs. F, C vs. $\mathrm{R}_{(t-2)}$, CPUE vs. N, F vs. f) se explican mediante modelos lineales. En todos los casos, dichos modelos resultaron significativos $(P<0.05)$ y la relación entre las dos variables de cada modelo es positiva (Cuadro 2).

La mayoría de los modelos que relacionan los pares de variables tienen un mejor ajuste a los datos con las estimaciones obtenidas según el modelo VPA. Los
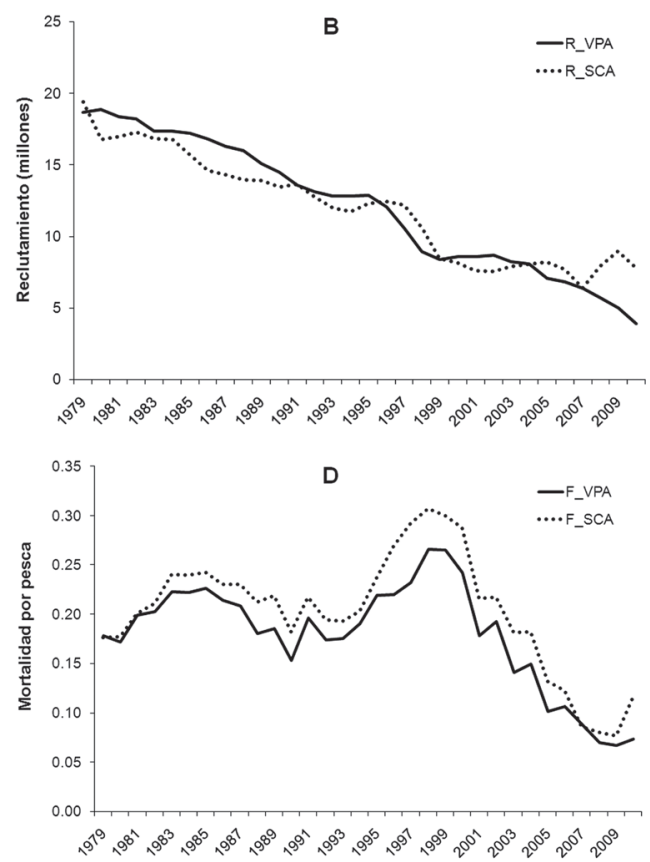

Fig. 3. Series anuales de las variables estimadas según los modelos análisis de población virtual (VPA) y análisis estadístico de captura por edades (SCA) para la población de langosta espinosa (Panulirus argus) en la región suroriental de Cuba. A) Tamaño total, número de langostas (N). B) Reclutamiento, i.e., número de langostas con 1 año de edad (R). C) Biomasa total en toneladas (B). D) Tasa de mortalidad por pesca (F)

Fig. 3. Annual series of estimated variables according to the Virtual Population Analysis (VPA) and the Statistical Catch-at-Age Analysis (SCA) models for the spiny lobster (Panulirus argus) population in Cuba's southeastern region. A) Total size, number of lobsters (N). B) Recruitment, i.e., number of lobsters with 1 year of age (R). C) Total biomass in tons (B). D) Fishing mortality rate (F) 
Cuadro 1. Media, desviación estándar (SD) y tamaño de muestra (n) de todas las variables según ambos modelos (VPA y SCA) para la población de langosta (Panulirus argus) en la región suroriental de Cuba. Tamaño total de la población en millones de individuos $(\mathrm{N})$, reclutamiento en millones de individuos $(\mathrm{R})$, biomasa total en toneladas (B) y tasa de mortalidad por pesca (F)

Table 1. Mean, standard deviation (SD) and sample size (n) of all variables according to both models (VPA and SCA) for the spiny lobster (Panulirus argus) population in Cuba's southeastern region. Total population size in millions of individuals $(\mathrm{N})$, recruitment in millions of individuals (R), total biomass in tons (B) and fishing mortality rate (F)

\begin{tabular}{cccc}
\hline Variable & $\mathbf{n}$ & $\begin{array}{c}\text { VPA } \\
\text { Media } \pm \text { SD }\end{array}$ & $\begin{array}{c}\text { SCA } \\
\text { Media } \pm \text { SD }\end{array}$ \\
\hline N & 32 & $35.428 \pm 11.713$ & $34.201 \pm 10.024$ \\
R & 32 & $12.076 \pm 4.612$ & $11.939 \pm 3.697$ \\
B & 32 & $11634.4 \pm 3003.2$ & $10837.0 \pm 2905.5$ \\
F & 32 & $0.18 \pm 0.05$ & $0.20 \pm 0.06$ \\
\hline
\end{tabular}

Cuadro 2. Relaciones entre las variables estimadas según los modelos VPA y SCA para la población de langosta (Panulirus argus) en la región suroriental de Cuba. Captura (C), captura por unidad de esfuerzo (CPUE), esfuerzo pesquero (f), tamaño total de la población $(\mathrm{N})$, reclutamiento de dos años anteriores $\left(\mathrm{R}_{\mathrm{t}-2}\right)(\mathrm{t}$-año), biomasa total $(\mathrm{B})$ y tasa de mortalidad por pesca $(\mathrm{F})$. Criterio de Información de Akaike corregido $\left(\mathrm{AIC}_{\mathrm{C}}\right)$. Nivel de apoyo empírico del modelo $\left(\Delta_{i}\right)$ : a menor valor mejor ajuste del modelo. Todas las variables se transformaron con logaritmo. Se señala en negrita el modelo con mejor ajuste para cada relación

Table 2. Relationships between estimated variables by the VPA and SCA models for the spiny lobster (Panulirus argus) population in the southeastern region of Cuba. Catch (C), catch per unit effort (CPUE), fishing effort (f), total population size (N), recruitment from two previous years $\left(\mathrm{R}_{\mathrm{t}-2}\right)$ ( $\mathrm{t}$-years), total biomass $(\mathrm{B})$ and fishing mortality rate $(\mathrm{F})$. Akaike Information Criterion $\left(\mathrm{AIC}_{\mathrm{C}}\right)$. Model's empirical support level $\left(\Delta_{i}\right)$ : the lower the value the better the fit. All variables were transformed with logarithm. The bestfitting model for each relationship is highlighted in bold

\begin{tabular}{lcrrrccccrr}
\hline \multicolumn{1}{c}{ VPA } & \multicolumn{1}{c}{ SCA } \\
\hline $\begin{array}{c}\text { Relación entre } \\
\text { las variables }\end{array}$ & $\mathbf{R}^{2}$ & $\mathbf{F}(\mathbf{1}, \mathbf{3 0})$ & $\mathbf{A I C}_{\mathbf{c}}$ & $\boldsymbol{\Delta}_{\boldsymbol{i}}$ & $\begin{array}{c}\text { Relación entre } \\
\text { las variables }\end{array}$ & $\mathbf{R}^{2}$ & $\mathbf{F}(\mathbf{1}, \mathbf{3 0})$ & $\mathbf{A I C}_{\mathrm{c}}$ & $\boldsymbol{\Delta}_{\boldsymbol{i}}$ \\
\hline $\mathrm{C}=1.303 \mathrm{~N}-2.655$ & 0.83 & 141.89 & 73.51 & $\mathbf{0}$ & $\mathrm{C}=1.443 \mathrm{~N}-3.278$ & 0.71 & 74.40 & 89.47 & 15.96 \\
$\mathrm{C}=1.608 \mathrm{~B}-3.275$ & 0.71 & 74.40 & 89.43 & $\mathbf{0}$ & $\mathrm{C}=1.463 \mathrm{~B}-2.642$ & 0.61 & 47.16 & 99.14 & 9.71 \\
$\mathrm{C}=1.215 \mathrm{~F}+4.178$ & 0.80 & 118.25 & 78.43 & $\mathbf{0}$ & $\mathrm{C}=1.169 \mathrm{~F}+4.077$ & 0.67 & 60.96 & 93.88 & 15.45 \\
$\mathrm{C}=1.353 \mathrm{R}_{(\mathrm{t}-2)}-2.279$ & 0.88 & 209.59 & 55.22 & $\mathbf{0}$ & $\mathrm{C}=1.552 \mathrm{R}_{(\mathrm{t}-2)}-3.076$ & 0.87 & 180.34 & 59.16 & 3.94 \\
$\mathrm{CPUE}=0.624 \mathrm{~N}-0.477$ & 0.66 & 58.17 & 54.92 & 3.9 & $\mathrm{CPUE}=0.765 \mathrm{~N}-1.111$ & 0.70 & 69.61 & 51.02 & $\mathbf{0}$ \\
$\mathrm{F}=1.002 \mathrm{f}-4.676$ & 0.93 & 391.75 & 25.07 & $\mathbf{0}$ & $\mathrm{F}=0.936 \mathrm{f}-4.361$ & 0.89 & 246.2 & 35.54 & 10.47 \\
\hline
\end{tabular}


valores del Índice de Akaike (AIC) fueron menores en todos los casos para el VPA que para el SCA, con excepción de la relación entre la captura por unidad de esfuerzo (CPUE) y el tamaño total de la población (N). El modelo que relaciona a estas dos variables se ajusta mejor a las estimaciones obtenidas con el modelo SCA (Cuadro 2).

\section{DISCUSIÓN}

Según Baisre (2000), la explotación pesquera de la langosta ( $P$. argus) en Cuba ha pasado por las fases típicas de desarrollo de una pesquería, descritas por Caddy (1984): fase de predesarrollo, fase de crecimiento, fase completamente explotada, fase de sobreexplotación, fase de colapso y fase de recuperación. La pesquería en la región suroriental de Cuba debe encontrarse en esta última fase, pero, a pesar del perfeccionamiento del sistema de manejo de la pesquería en el país y de la consecuente disminución del esfuerzo pesquero desde el año 2000, no se observan signos de recuperación en las capturas de esta zona.

Al ser considerada la tasa de mortalidad natural $\left(\mathrm{M}=0.34 \mathrm{año}^{-1}\right)$ como un valor constante para todo el período analizado (1979-2010), se puede considerar que el grado de explotación (definido por Gulland (1971) E = F / Z, donde E es la tasa de explotación, $\mathrm{F}$ es la tasa de mortalidad por pesca y Z es la tasa instantánea de mortalidad total) depende en forma directa de la tasa de mortalidad por pesca, por lo cual está estrechamente relacionado con las características del esfuerzo pesquero, demostrado por ambos modelos aplicados, por la alta relación existente entre estas dos variables $\left(R^{2}{ }_{\text {VPA }}=0.93, R^{2}\right.$ $\left.{ }_{\mathrm{SCA}}=0.89\right)$. Otros autores han encontrado elevados coeficientes de determinación entre la tasa de mortalidad por pesca y el esfuerzo pesquero, como en el Golfo de Batabanó, región suroccidental de Cuba $\left(\mathrm{R}^{2}=0.79\right.$, Puga et al. 2005).
Según los resultados obtenidos por los dos modelos analíticos aplicados [análisis de población virtual (VPA) y análisis estadístico de captura por edades (SCA)], el tamaño de la población de langostas (N) y el reclutamiento de langostas con un año de edad a la pesquería $(\mathrm{R})$ han disminuido en los últimos años y no se observa una recuperación, a pesar de la continua disminución de la tasa de mortalidad por pesca (F) aplicada en la zona desde el año 1999. Aunque el modelo SCA muestra una estabilización de estas variables en la última década.

Las reducciones en el reclutamiento se han observado en otras poblaciones de langostas, llegando a ser hasta del $50 \%$ en los casos de P. argus en Florida (Muller et al. 2000) y Brasil (Ehrhardt \& Sobreira, 2003), P. cygnus en Australia Occidental (Caputi et al. 2001) y P. marginatus en Hawai (Polovina et al. 1995). En general, el reclutamiento ha sido afectado por la intensa explotación en muchos países del Caribe, lo cual, unido a factores climáticos, enfermedades en juveniles y afectaciones del hábitat por la actividad humana, han causado disminuciones de la abundancia y, por lo tanto, de las capturas del recurso a escala regional (Ehrhardt et al. 2011).

La biomasa total de la población de langostas (B) muestra una similar tendencia a la disminución que el tamaño poblacional (N) y que el reclutamiento (R). Pero en este caso ambos modelos muestran en la última década una tendencia a la estabilización de la biomasa, lo cual puede relacionarse con el comportamiento del índice de abundancia relativa empleado, captura por unidad de esfuerzo (CPUE) que ha mostrado una tendencia similar en esta última década. Según Punt et al. (2002), quienes evaluaron varios modelos aplicados a diferentes especies comerciales, el modelo SCA proporciona con mayor precisión las estimaciones de biomasa y, en general, fue considerado como el mejor método a aplicar para esas especies. 
Al relacionar las variables estimadas con las variables observadas (C vs. N, C vs. $\mathrm{B}, \mathrm{C} v s . \mathrm{F}, \mathrm{C} v s . \mathrm{R}_{(\mathrm{t}-2)}$, CPUE vs. N, F vs. f), se ajustaron significativamente a modelos lineales. Aunque se pueden intentar múltiples relaciones entre pares de variables, estas fueron escogidas siguiendo un criterio lógico de causa-efecto, donde las capturas dependen de las variables poblacionales estimadas (N, R, B), así como de la mortalidad por pesca $(\mathrm{F})$. Cabe destacar que, en la práctica, la relación estadística per se y los datos tomados en el presente estudio no justifican una relación de causalidad, de ahí que los resultados de las regresiones se deban interpretar con precaución.

Entre todas las variables relacionadas con la captura, el mayor índice de determinación se encontró para la relación propuesta entre $\mathrm{C} \mathrm{y} \mathrm{R}_{(\mathrm{t}-2)}$. Esto sugiere que de todas las variables consideradas, la de mayor influencia sobre la captura esperada es el reclutamiento de langostas con un año de edad a la pesquería con un desfase de dos años. Esto ha sido reportado por otros autores para la pesquería de $P$. argus en Cuba, Florida y Nicaragua-Honduras (Ehrhardt et al. 2011; Puga et al. 2010).

Para la mayoría de las relaciones, según los valores de $\Delta_{i}$, hay un mejor ajuste para las variables calculadas por el modelo VPA, por lo cual se puede considerar a este modelo como más adecuado para los datos de la pesquería de langosta en esta región. Aunque la consideración más importante es que los modelos utilizados deben ser coherentes con los datos disponibles (Butterworth \& Rademeyer, 2008).

Ambos modelos se encuentran entre las alternativas más comúnmente utilizadas para conducir los modelos de evaluación de stocks de poblaciones estructuradas en la edad. Sus requerimientos mínimos de datos son: las capturas comerciales, el número de individuos capturados en cada edad y el esfuerzo o algún índice de abundancia relativa a través del tiempo.
La primera alternativa, el modelo VPA, depende de la idea de que al tener registros de la captura por edades de un grupo de cohortes hasta que todas las cohortes han muerto, entonces debe poderse calcular hacia atrás, literalmente, el número de individuos por edades que debió haber existido. De este modo, el tamaño de la población puede ser proyectado de atrás hacia adelante, a través del tiempo y de las edades hasta que se obtengan estimados de los reclutamientos originales (Megrey, 1989).

La segunda alternativa, el modelo SCA, se puede considerar como el reverso de la primera, proyectando las cohortes hacia adelante, a través del tiempo y de las edades (Haddon, 2001).

La principal desventaja de un método sobre el otro se refiere a que los requerimientos de datos para un VPA son muy estrictos, ya que no puede haber años de información perdida para que los cálculos puedan realizarse de manera ininterrumpida. El modelo SCA puede correr con espacios vacíos en la información (Butterworth \& Rademeyer, 2008; Haddon, 2001). Este hecho, de ser menos estricto en sus requerimientos de datos, significa que es un método más práctico para muchas pesquerías en países sin una larga tradición de recolectar información estructurada por edad (Haddon, 2001).

Teniendo en cuenta que todos los modelos no son más que aproximaciones de la realidad, es necesario elegir los mejores modelos después de aplicados, ya que proporcionan diferentes perspectivas de un mismo problema. Aunque la principal ventaja es mantener la misma metodología a través del tiempo que permite mantener el statu quo (Butterworth \& Rademeyer, 2008).

No obstante, los modelos comparten muchas de sus ecuaciones esenciales, por lo que el uso de cada uno debe depender de la disponibilidad de datos en las pesquerías y de la finalidad con la que se aplique cada modelo. En la presente investigación 
se propone el uso de los criterios de información como una herramienta de apoyo a la aplicación de los modelos en los estudios de pesquería de langostas.

\section{CONCLUSIONES}

El tamaño de la población de langostas y el reclutamiento de langostas con un año de edad a la pesquería han disminuido en los últimos años y no se observa una recuperación, a pesar de la continua disminución de la tasa de mortalidad por pesca aplicada en la zona desde el año 1999. Aunque el análisis estadístico de captura por edades (SCA) muestra una estabilización de estas variables ( $\mathrm{N}$ y $\mathrm{R}$ ) en la última década; la biomasa total de la población de langostas (B) muestra una similar tendencia a la disminución, pero en este caso ambos modelos aplicados expresan en la última década una tendencia a la estabilización. La captura presenta una mayor relación con el reclutamiento de langostas con un año de edad a la pesquería de dos años antes. Siguiendo los valores de $\Delta_{i}$, el análisis de población virtual (VPA) garantiza un mejor ajuste a relaciones lineales entre las variables estimadas y los datos de la pesquería de langosta en la región suroriental de Cuba.

\section{BIBLIOGRAFÍA}

Alzugaray, R. \& Puga, R. (2010). Dinámica poblacional de la langosta Panulirus argus (Latreille, 1804) en la región suroriental de Cuba. Rev. Cub. Invest. Pesq., 27(1), 1-6.

Arce, A. M. \& de León, M. E. (2001). Biology. En P. Medley \& S. Venema (Eds.), Report on the FAO/DANIDA/ CFRAMP/WECAFC Regional Workshops on the Assessment of the Caribbean Spiny Lobster (Panulirus argus) (pp. 17-25). FAO Fisheries Reports No. 619. Rome, Italy: FAO.

Baisre, J. A. (2000). The Cuban spiny lobster fishery. En B. F. Phillips \& J. Kittaka (Eds.), Spiny Lobsters Fisheries and Culture (pp. 135-154). Oxford, England: Fishing News Books.

Burnham, K. P. \& Anderson, D. R. (2002). Model Selection and Multimodel Inference: A Practical Information-Theoretic Approach, Second. New York, EE.UU.: Springer-Verlag.

Butterworth, D. S. \& Rademeyer, R. A. (2008). Statistical catch-at-age analysis vs ADAPT-VPA: the case of Gulf of Maine cod. ICES J. Mar. Sci., 65(9), 1717-1732.

Caddy, J. F. (1984). An alternative to equilibrium theory for fishery management. En FAO (Ed.), Papers presented at the Expert consultation on the regulation of fishing effort (fishing mortality), Rome, 17-26 Jan 1983 (pp. 173-214). FAO Fisheries Reports No. 289(2). Rome, Italy: FAO.

Caputi, N., Chubb, C. C. \& Pearce, A. (2001). Environmental effects on recruitment on the western rock lobster, Panulirus cygnus. Mar. Freshwat. Res., 52(8), 1167-1174.

Cruz, R., Baisre, J. A., Díaz, E., Brito, R., García, C., Blanco, W. \& Carrodeguas, C. (1990). Atlas Biológico-Pesquero de la Langosta en el Archipiélago Cubano. Publicación Especial Revista Cubana de Investigaciones Pesqueras y Revista Mar y Pesca. La Habana, Cuba: MIP.

Cruz, R., Coyula, R. \& Ramírez, A. T. (1981). Crecimiento y mortalidad de la langosta espinosa (Panulirus argus) en la plataforma suroccidental de Cuba. Rev. Cub. Investig. Pesq., 6(4), 89-119. 
Cruz, R. \& de León, M. E. (1991). Dinámica reproductiva de la langosta $(\mathrm{Pa}$ nulirus argus) en el archipiélago cubano. Rev. Invest. Mar., 12(1-3), 234-245. de León, M. E., Cruz, R. \& Puga, R. (1995). Actualización de la edad y el crecimiento de la langosta espinosa Panulirus argus. Rev. Cub. Invest. Pesq., 19(2), 3-8.

de León, M. E., Puga, R. \& Cruz, R. (1991). Panorama de la pesquería de langosta en Cuba durante 1989. Rev. Cub. Invest. Pesq., 16(3-4), 21-29.

Ehrhardt, N. M. (2001). Regional Review. En P. Medley \& S. Venema (Eds.), Report on the FAO/DANIDA/CFRAMP/ WECAFC Regional Workshops on the Assessment of the Caribbean Spiny Lobster (Panulirus argus) (pp. 1216). FAO Fisheries Reports No. 619. Rome, Italy: FAO.

Ehrhardt, N., Puga, R. \& Butler, M. J. IV (2011). Implications of the ecosystem approach to fisheries management in large ecosystems. The case of the $\mathrm{Ca}$ ribbean spiny lobster. En L. Fanning, R. Mahon \& P. McConney (Eds.), Towards Marine Ecosystem-Based Management in the Wider Caribbean (pp. 157-175). Amsterdam, Holanda: Amsterdam University Press.

Ehrhardt, N. M. \& Sobreira, C. A. (2003). An assessment of the Brazilian spiny lobster, Panulirus argus, fishery. FAO Fish. Rep., 715, 68-74.

Gavaris, S. (1988). An adaptive framework for the estimation of population size. Ottawa, Canada: Dept. of Fisheries and Oceans.

Gulland, J. A. (1971). The fish resources of the ocean. Surrey, England: Fishing News Books.
Haddon, M. (2001). Modelling and Quantitative Methods in Fisheries. London, England: Chapman \& Hall/CRC.

Hilborn, R. \& Walters, C. J. (1992). Quantitative fisheries stock assessment: Choice, dynamics and uncertainty. London, England: Chapman and Hall.

Jurado-Molina, J., Livingston, P. A. \& Ianelli, J. N. (2005). Incorporating predation interactions in a statistical catch-at-age model for a predator-prey system in the eastern Bering Sea. Can. J. Fish. Aquat. Sci., 62(8), 1865-1873.

Lassen, H. \& Medley, P. (2000). Virtual population analysis: a practical manual for stock assessment. FAO Fisheries Technical Papers No. 400. Rome, Italy: FAO.

Lipcius, R. N. (1985). Size-dependent reproduction and molting in spiny lobsters and other long-lived decapods. En W. A. Wenner (Ed.), Crustacean Issues, vol. 3. Factors in Adult Growth (pp. 129-148). Rotterdam, Holanda: Balkema.

MacCall, A. D. (1986). Virtual Population Analysis (VPA) equations for nonhomogeneous populations, and a family of approximations including improvements on Pope's cohort analysis. Can. J. Fish. Aquat. Sci., 43(12), 2406-2409.

Megrey, B. A. (1989). Review and comparison of age structured stock assessment models from theoretical and applied points of view. Am. Fish. Soc. Symp., 6, 8-48.

Muller, R. G., Sharp, W. C., Matthews, T. R., Bertelsen, R. \& Hunt, J. H. (2000). The 2000 update of the stock assessment for spiny lobster, Panulirus argus, in the Florida Keys. Florida, EE.UU.: Fish and Wildlife Conservation Commission. 
Polovina, J. J., Haight, W. R., Moffitt, R. B. \& Parrish, F. A. (1995). The role of benthic habitat, oceanography, and fishing on the population dynamics of the spiny lobster, Panulirus marginatus (Decapoda, Palinuridae), in the Hawaiian Archipelago. Crustaceana, 68(2), 203-212.

Puga, R., Hernández-Vázquez, S., LópezMartínez, J. \& de León, M. E. (2005). Bioeconomic modelling and risk assessment of the Cuban fishery for spiny lobster Panulirus argus. Fish. Res., 75(1-3), 149-163.

Puga, R., Piñeiro, R., Cobas, S., de León, M. E., Capetillo, N. \& Alzugaray, R. (2010). La pesquería de la langosta espinosa, conectividad y cambio climático en Cuba. En A. Hernández-Zanuy y P. M. Alcolado (Eds.), La biodiversidad en ecosistemas marinos y costeros del litoral de Iberoamérica y el cambio climático: I. Memorias del Primer
Taller de la Red CYTED BIODIVMAR, La Habana, julio, 2010 (CD-ROM, pp. 112-131). La Habana, Cuba: Instituto de Oceanología.

Punt, A. E., Smith, A. D. M. \& Cui, G. (2002). Evaluation of management tools for Australia's South East Fishery 2. How well do commonly-used stock assessment methods perform? Mar. Freshwat. Res., 53, 631-644.

Quinn, G. P. \& Keough, M. J. (2002). Experimental Design and Data Analysis for Biologist. Cambridge, EE.UU.: Cambridge University Press.

Sparre, P. \& Venema, S. C. (1995). Introducción a la evaluación de recursos pesqueros tropicales. Parte 1. Manual. FAO Documento Técnico de Pesca No. 306.1. Valparaíso, Chile: Editorial Universitaria.

StatSoft, Inc. (2001). STATISTICA (data analysis software system), version 6. www.statsoft.com. Tulsa, OK.: StatSoft, Inc. 